\title{
UMA REVISÃO DAS ÚLTIMAS INOVAÇÕES EM INSPEÇÕES DE ESTRUTURAS
}

\author{
SOLLERO, MARCELA BARROS DE SOUZA \\ Tecnóloga em Construção Civil \\ CONCREMAT Engenharia e Tecnologia S.A. \\ São Paulo; Brasil \\ marcela.barros@concremat.com.br
}

\author{
SANTOS, RODRIGO TADEU DOS \\ Engenheiro Civil \\ CONCREMAT Engenharia e Tecnologia S.A. \\ São Paulo; Brasil \\ rodrigo.tadeu@concremat.com.br
}

\section{RESUMO}

As estruturas, sejam elas de concreto armado ou metálicas, sofrem a ação de mecanismos de deterioração ao longo dos anos. Como consequência, sua durabilidade, sua funcionalidade e sua estabilidade podem ser comprometidas pela ocorrência de manifestações patológicas, cuja deteç̧ão precoce proporciona a redução de custos de manutenção e de paradas não programadas. A inspeção visual de estruturas constitui uma etapa essencial para o diagnóstico do quadro patológico instaurado, fundamentando planos de manutenção e projetos de recuperação e reforço. Ao longo dos últimos anos, diversas técnicas vem sendo adaptadas e desenvolvidas para tornar as inspeções mais rápidas, seguras e abrangentes, fazendo uso de recursos como drones, escaneamento a laser $3 \mathrm{D}$, fotogrametria e tomografias. O presente artigo apresenta um panorama das inovações atualmente empregadas na inspeção de estruturas em diversos países, bem como casos de aplicação em obras de arte especiais e áreas industriais, discutindo-se a aplicabilidade das técnicas e recursos de análise de dados - big data.

Palavras-chave: inspeção, inovação, escaneamento a laser 3D, VANT, drone.

\section{ABSTRACT}

Structures, whether concrete or metallic, are prone to the action of deterioration mechanisms over the years. As a consequence, their durability, stability, and functionality can be compromised by the occurrence of pathological manifestations, which early detection reduces maintenance costs and unscheduled downtime reduction. The visual inspection of structures is a fundamental step for the diagnosis of the established pathological condition, supporting maintenance plans and recovery and reinforcement projects. Over the past few years, many techniques have been adapted and developed to make inspections faster, safer and more comprehensive, using assets such as drones, 3D laser scanning, photogrammetry, and CT scans. This paper presents an overview of currently applied innovations in structure inspection in many countries, as well as application cases, discussing the applicability of data analysis resources and techniques - big data.

Keywords: inspection, innovation, 3D laser scanning, LiDAR, UAS, drone.

\section{INTRODUÇÃO}

As estruturas, sejam elas de concreto armado ou metálicas, estão sujeitas a mecanismos de degradação ao longo do tempo. Entre eles, pode-se destacar a corrosão do aço, os ataques químicos, o desgaste, a exposição térmica, as reações expansivas e a lixiviação (CEN, 2008; ABNT, 2014). A ação desses mecanismos tende a afetar os aspectos da durabilidade, da funcionalidade e da estabilidade das estruturas, podendo reduzir sua vida útil e até mesmo propiciar acidentes, como a queda de materiais ou seu colapso.

A norma ABNT NBR 5674:2012 (ABNT, 2012) defende que "é inviável, do ponto de vista econômico, e inaceitável, sob o ponto de vista ambiental, considerar as edificações como produtos descartáveis, passíveis da simples substituição por novas construções quando os requisitos de desempenho atingem níveis inferiores àqueles exigidos". As estruturas, portanto, devem ser alvo de manutenções períodicas de forma a manter seus requisitos de desempenho, considerando-se os mecanismos de degradação atuantes. 
Para que sejam realizadas intervenções de manutenção corretiva e preventiva para uma estrutura, é fundamental sua inspeção periódica e, na ocorrência de ações excepcionais, sua inspeção detalhada, especial ou extraordinária. A periodicidade da inspeção deve constar em seu Manual de Uso, Operação e Manutenção, quando existente; adicionalmente, ela pode ser definida por norma - como a ABNT NBR 9452:2019 - ou lei, sendo indicado o respeito ao critério mais rigoroso aplicável à estrutura em estudo (ABNT, 2012; 2019).

A inspeção das estruturas pode apresentar desafios quanto ao acesso, à exposição do inspetor a riscos, ao registro de dados para formação de histórico e à velocidade requerida. A Figura 1 ilustra a realização de inspeções em algumas dessas situações.
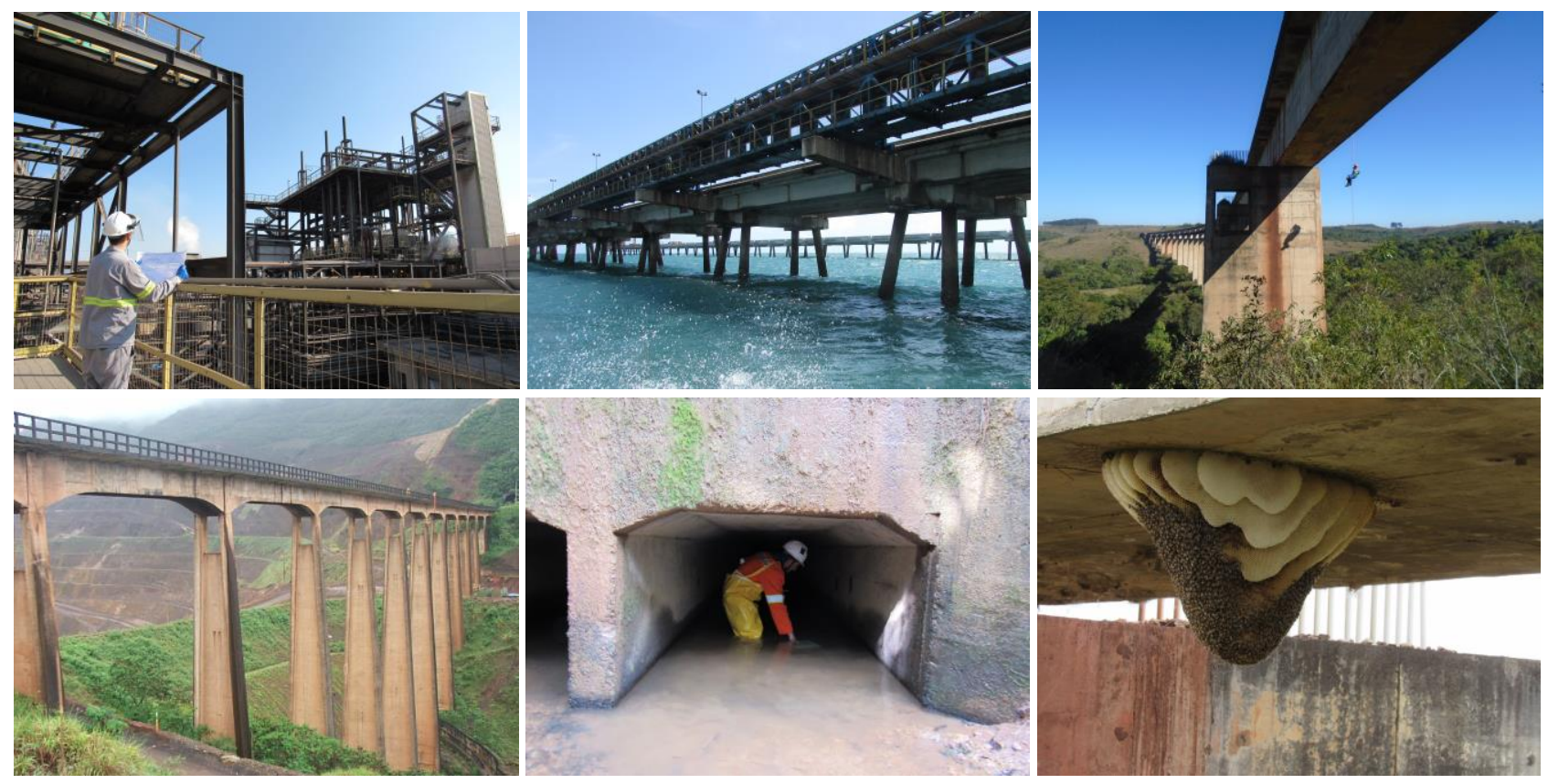

Figura 1: Inspeções em condições adversas

O uso de tecnologias inovadoras vem ao encontro desses desafios, permitindo a transferência de parte das inspeções a ambientes controlados, através de vídeos e ortofotos de alta resolução, da digitalização tridimensional e da aplicação de recursos como câmeras térmicas ou dotadas de infravermelho. O presente artigo apresenta e discute algumas dessas tecnologias.

\section{TECNOLOGIAS INOVADORAS EM INSPEÇÕES}

\subsection{REGISTRO DE IMAGENS E VÍDEOS COM VANTS OU DRONES}

O uso de veículos aéreos não tripulados (VANTs, também conhecidos como drones) equipados com câmeras e/ou sensores para a inspeção de pontes vem sendo amplamente estudado nos últimos anos; Duque (2017) cita a investigação prática dessa aplicação nos Estados Unidos desde 2007, relatando as dificuldades ocasionadas pela duração da bateria, pelas condições de iluminação e climáticas, pelo alcance do equipamento, pelo risco de colisão e pelas exigências dos orgãos reguladores. A Figura 2 ilustra estudos em campo realizados pelo autor. 

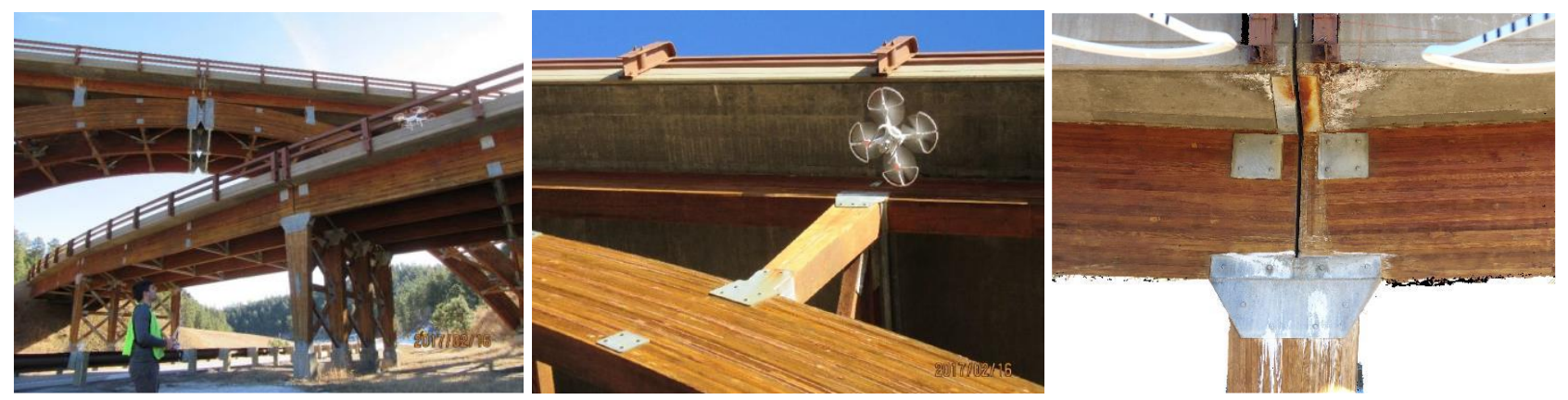

Figura 2: Inspeção de ponte com VANT. Adaptado de Duque (2017)

Em 2018, verificou-se que 70\% dos Departamentos de Transportes estaduais norte-americanos utilizavam VANTs rotineiramente e que $29 \%$ desses - o equivalente a 10 departamentos - aplicam o equipamento na inspeção de pontes e 17\%, na inspeção de pavimentos (AASHTO, 2018 apud FHWA, 2019).

Duque (2017) aponta que os resultados iniciais do uso de VANTs em inspeções de pontes por Departamento de Transporte estaduais norte-americanos se mostraram satisfatórios, foram obtidos mais rápidos do que se realizada uma inspeção convencional e permitiram o alcance a áreas de acessibilidade restrita. Corroborando o ponto de vista registrado por Duque (2017), o uso VANTs na inspeção de estruturas no Brasil vem se tornando mais frequente nos últimos anos.

A Figura 3 ilustra imagens obtidos na inspeção de um viaduto utilizando VANTs.

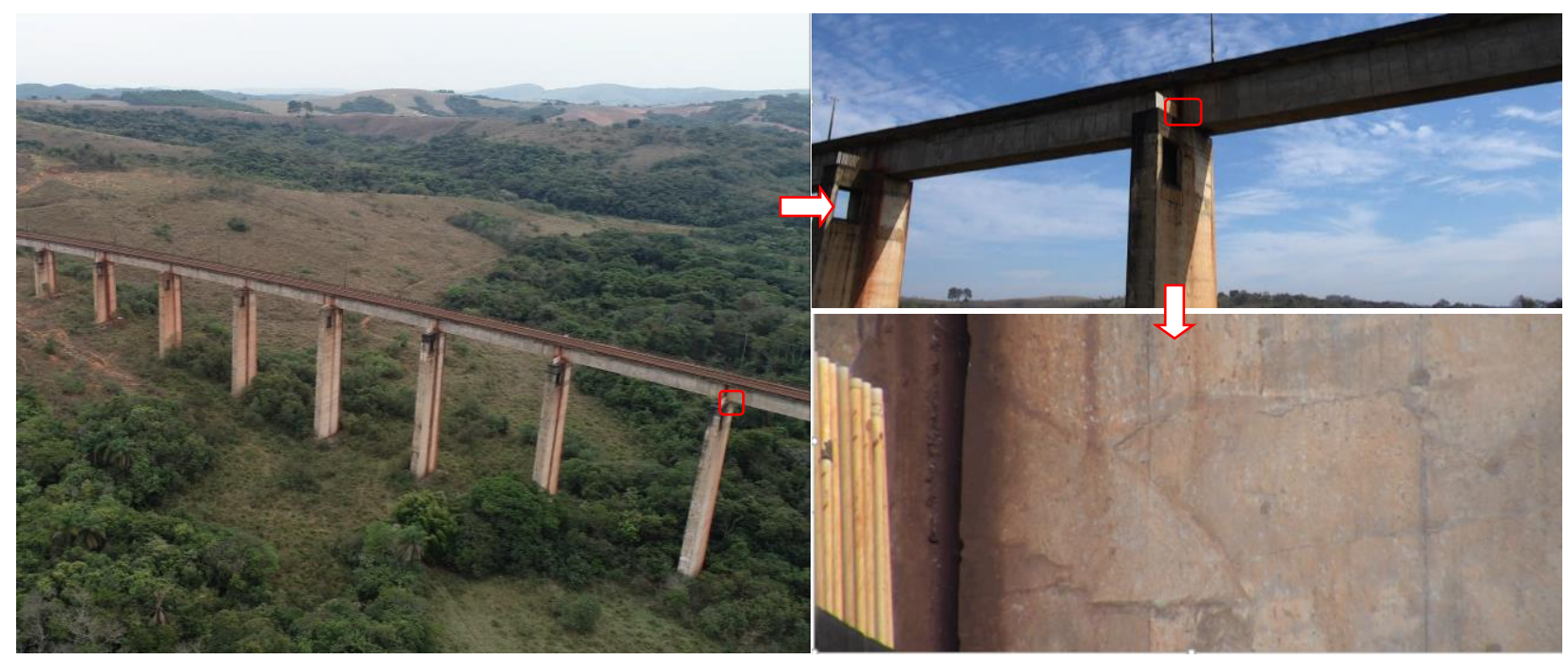

Figura 3: Inspeção de ponte com VANT, evidenciando a aproximação progressiva das imagens. Acervo dos autores.

No que se refere ao uso de softwares para detecção automatizada de anomalias, Duque (2017) constatou diferenças de 14,9 a 47,1\% na área de corrosão mapeada por técnicas computadorizadas de análise de imagem, em comparação com a inspeção tradicional; Kim et al (2018) desenvolveram um algoritmo aplicando conceitos de deep learning e redes neurais para a identificação de fissuras em estruturas de concreto, expondo que essa tecnologia permanece em seus estágios iniciais, mas é promissora. Como fatores limitantes, os autores destacam a necessidade de se manter o VANT a uma distância constante e conhecida da estrutura durante os trabalhos, as condições de luminosidade, o vento e a presença de manchas ou incrustações nos elementos analisados.

A Figura 4 ilustra a aplicação do algoritmo de Kim et al (2018) para detecção de fissuras no guarda-corpo de uma ponte. 

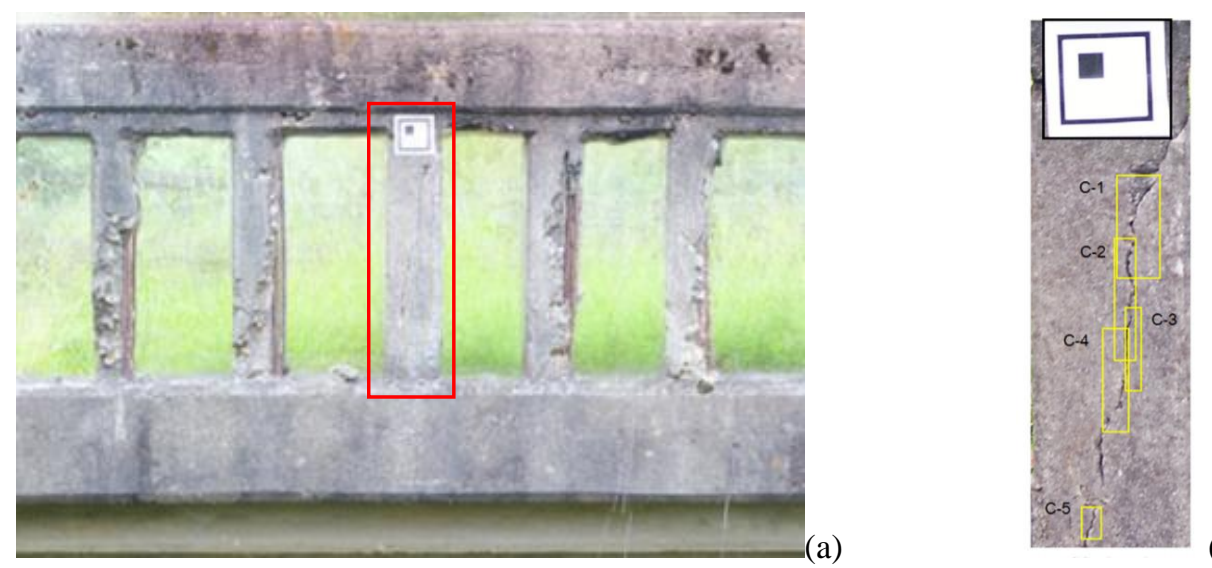

(b)

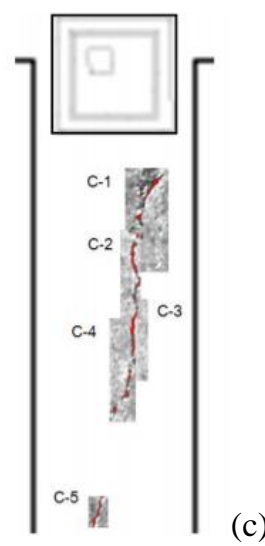

Figura 4: Detecção de fissuras em guarda-corpo por aplicação de algoritmo, adaptado de Kim et al (2018). A área analisada é assinalada em vermelho (a); a área ampliada é marcada em (b); o resultado é exposto em (c)

\subsection{ESCANEAMENTO A LASER 3D, FOTOGRAMETRIA E ESCANEAMENTO INFRAVERMELHO}

O escaneamento a laser 3D permite a elaboração de uma nuvem de pontos da estrutura analisada, que pode ser utilizada para a construção de modelos virtuais destinados ao uso em as builts, inspeções, monitoramentos ou análises estruturais. O resultado possui grande acurácia, proporcionando modelos de alta resolução, mas o tempo de processamento dos dados tende a ser longo e e o volume dos arquivos gerados é significativo.

A Figura 5 ilustra um viaduto junto a sua respectiva nuvem de pontos e ao modelo em elementos finitos elaborado com base na estrutura digitalizada.

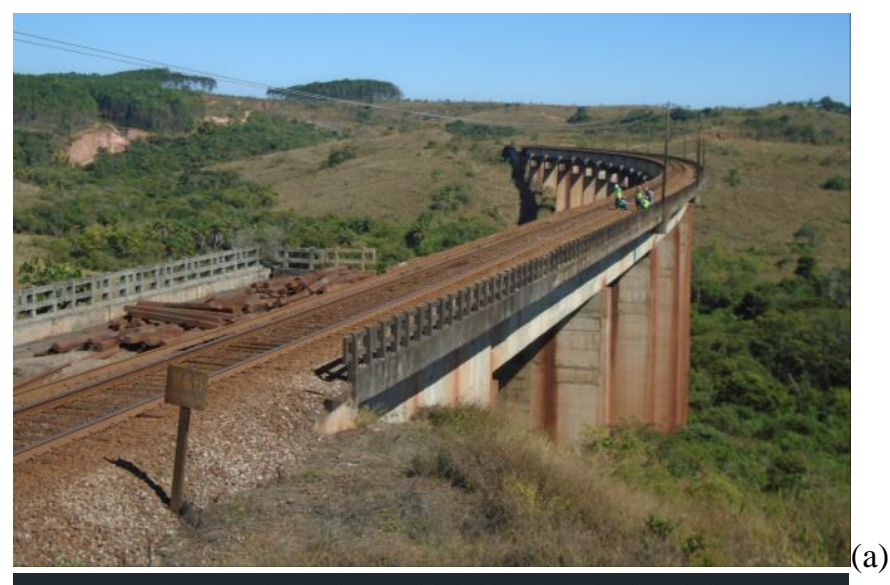

(a)
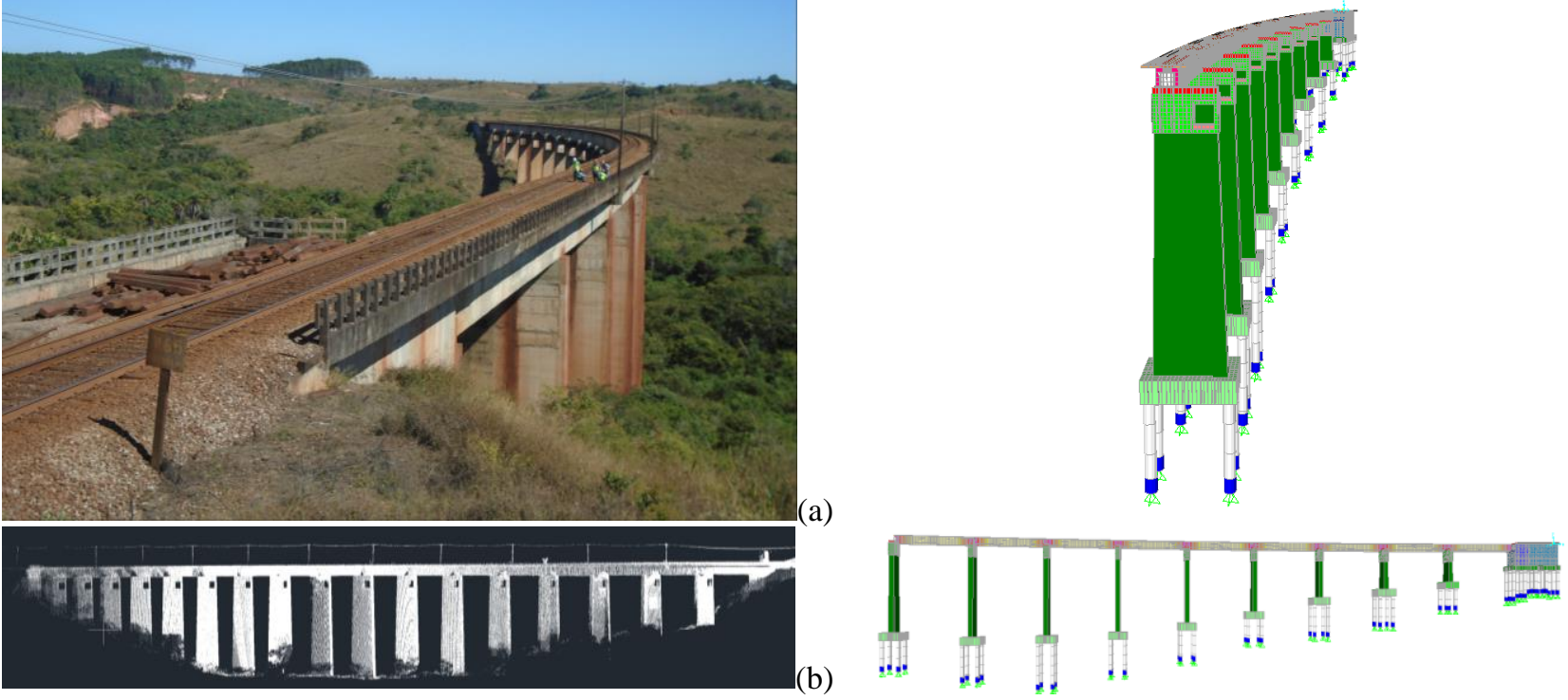

Figura 5: Vista do viaduto (a), nuvem de pontos da estrutura (b) e modelo em elementos finitos(c)

(c)

Meral (2011) aponta quatro fatores principais que podem afetar a acurácia de uma nuvem de pontos: calibração do equipamento, condições atmosféricas, propriedades do objeto e condições ambientais. Condições como a presença de partículas em suspensão, incrustações, vegetação, tráfego de veículos ou pedestres e obstruções também representam desafios à técnica; no que se refere a pontos de apoio para o equipamento, pode-se contornar sua escassez com o uso de VANTs.

Liu et al (2010) avaliaram a aplicação do escaneamento a laser de pontes na detecção de anomalias com dimensões superiores a 1 x $1 \mathrm{~cm}$ - o que exclui a maiora das fissuras, em função de sua abertura-, com base na alteração volumétrica proporcionada por desplacamentos, falhas de concretagem superficiais ou esborcinamentos. Para tal, os autores determinam uma superfície de referência plana e registram os gradientes verificados através do escaneamento; um algoritmo desenvolvido com esse propósito calcula o volume das regiões afetadas, como ilustra a Figura 6. 

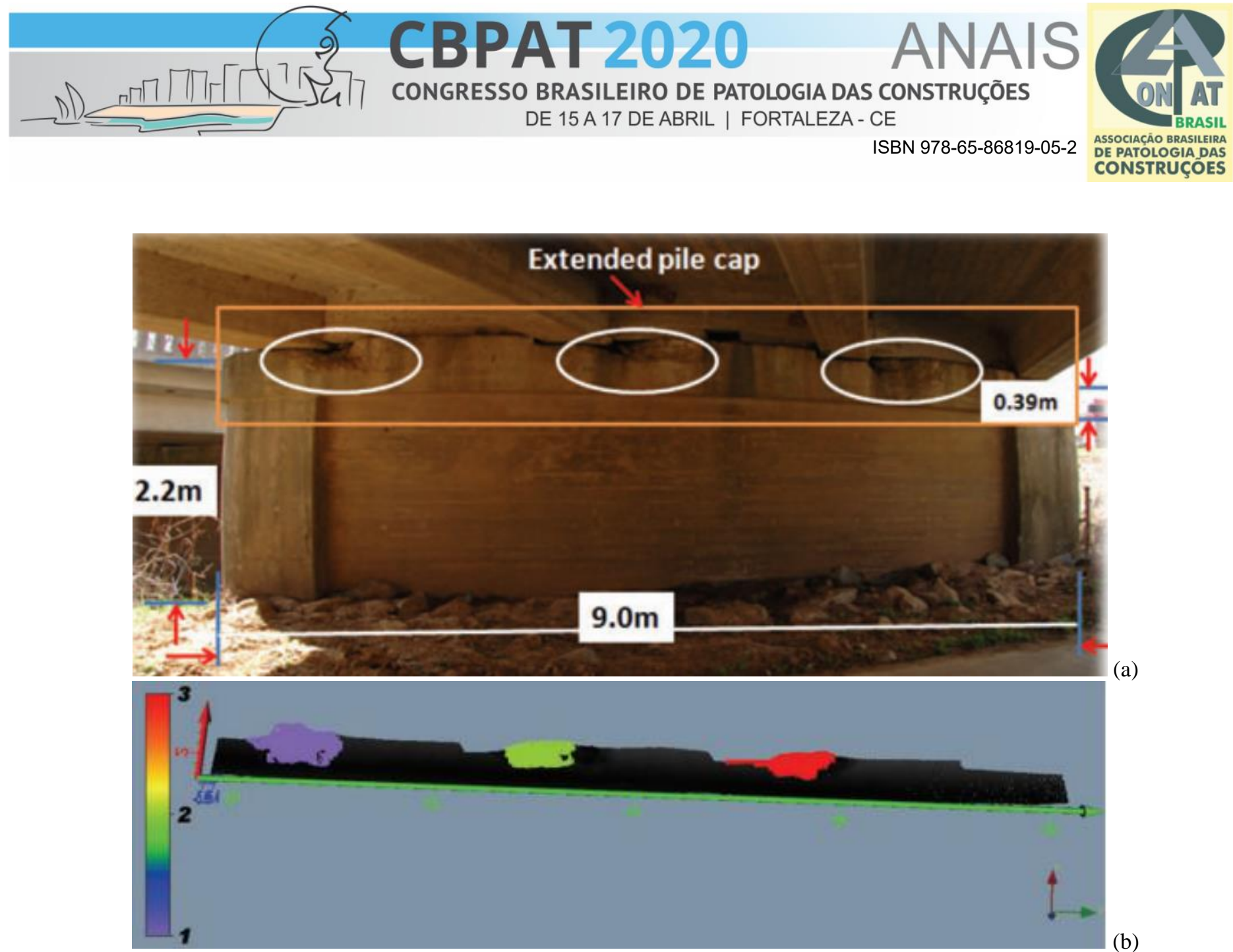

(a)

Figura 6: Vista da ponte, destacando-se os três pontos de concreto disgregado (a) e imagem demonstrando as anomalias localizadas e analisadas pelo algoritmo (b). Adaptado de Liu et al (2010)

Entre outras técnicas que podem ser utilizadas, em conjunto com o escaneamento a laser 3D, encontram-se o escaneamento infravermelho, muito semelhante a ele, e a fotogrametria. A fotogrametria pode ser definidida como a ciência e arte de se obter medidas confiáveis de fotografias, eliminando-se distorções. Através dela, podem ser geradas ortofotos, em que há uma sobreposição parcial das imagens em escala, assim como modelos 3D.

A Figura 7 apresenta a ortofoto da cobertura de um galpão industrial no Brasil, junto a um detalhe obtido por drone; as técnicas foram utilizadas em conjunto para o mapeamento de anomalias, eliminando a necessidade de trabalho em altura na inspeção, conforme as especificações do proprietário da estrutura.
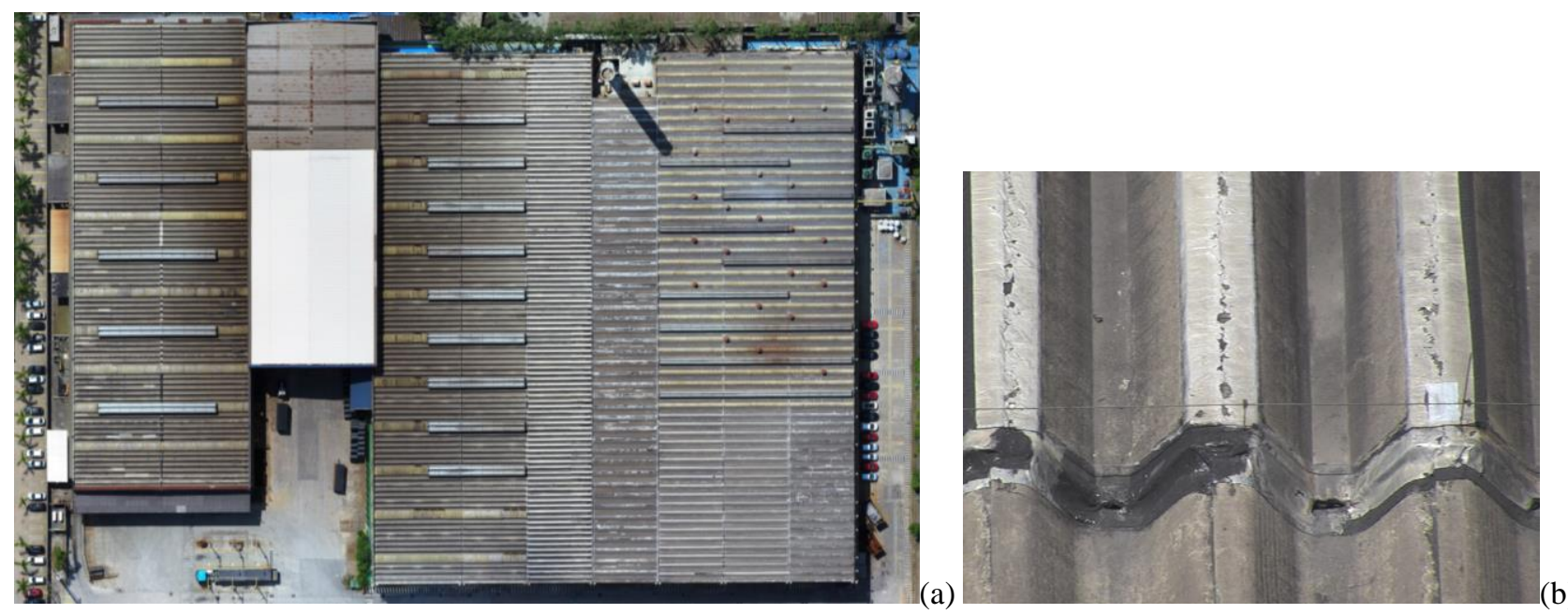

Figura 7: Ortofoto da cobertura de um galpão (a) e detalhe da cobertura (d). Acervo dos autores. 
Popescu et al (2019) avaliaram o uso das técnicas de escaneamento com laser terreste, fotogrametria de curta distância e escaneamento infravermelho em pontes suecas, como ilustra a Figura 8.
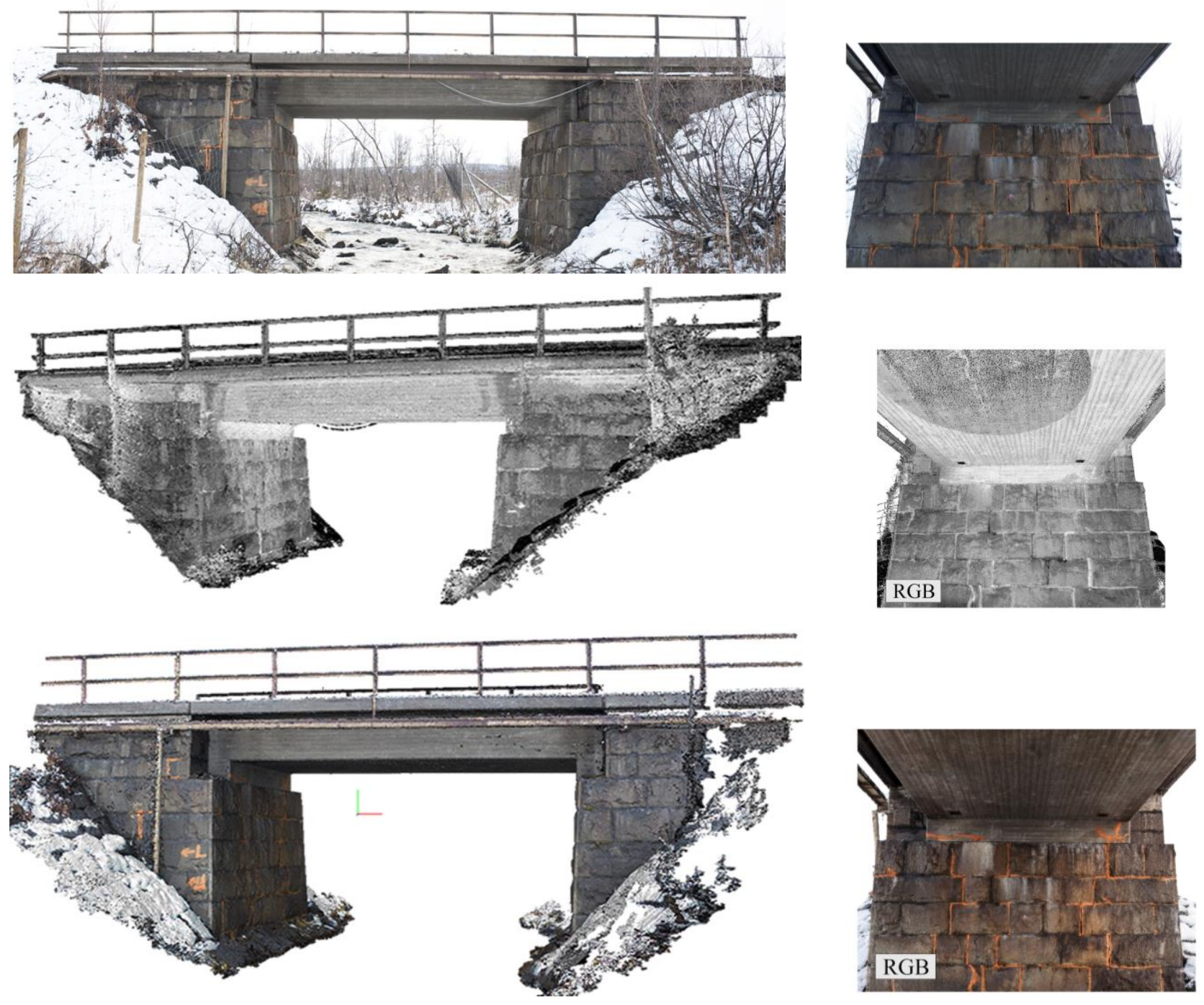

(c)
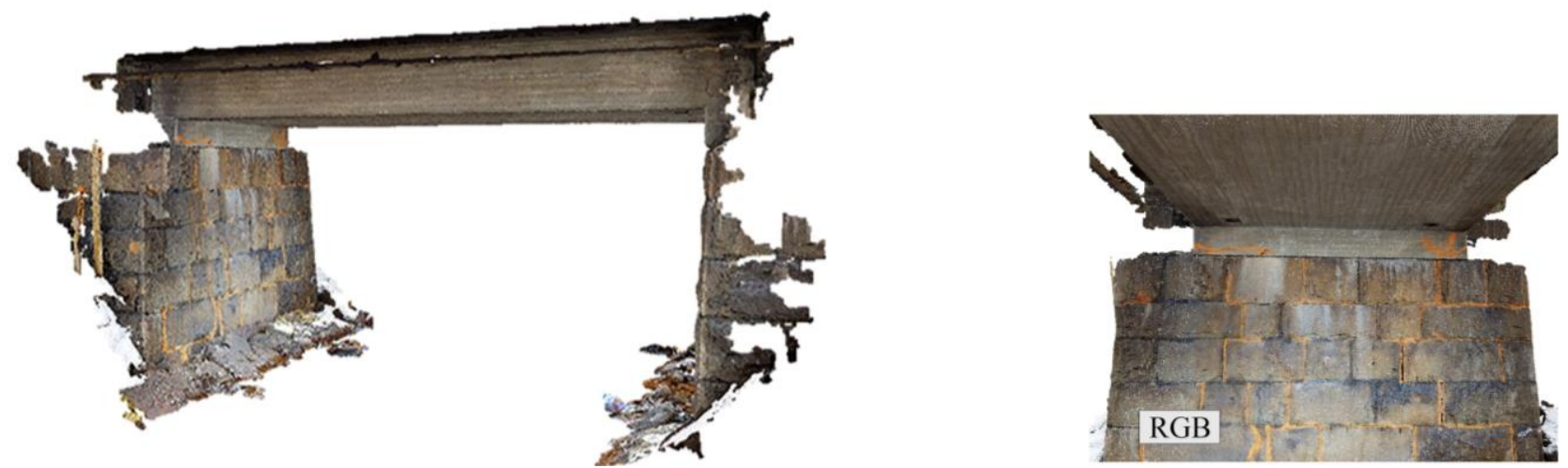

Figura 8: Fotografias de uma ponte (a) e modelos 3D obtidos por escaneamento com laser terreste (b), fotogrametria (c) e escaneamento infravermelho (d). Adaptado de Popescu et al (2019)

Foram realizados 11 posicionamentos do scanner a laser terreste com 30 a 40 milhões de ponto por escaneamento, 744 fotos com ISO 160 e 67 posicionamentos do scanner infravermelho. Para a fotogrametria, utilizaram-se câmeras digitais; foram fixados alvos e barras de calibração nas estruturas e as fotos possuíram no mínimo 60\% de sobreposição transversal e longitudinal. Para o escaneamento infravermelho, utilizaram-se câmeras RGB-D, câmeras infravermelhas e um projetor infravermelho; dada a alta sensibilidade da técnica à luz, as imagens foram registradas no crepúsculo. 
Observa-se que a nuvem derivada da fotogrametria é mais realista do que a obtida por outras técnicas. Na Tabela 1 , no entanto, observa-se que a nuvem de pontos obtida por escaneamento a laser possui a maior acurácia (POPESCU et al, 2019). Ainda que o exemplo apresentado compare as dimensões dos elementos, a mesma lógica pode ser aplicada para a medição de anomalias registradas na estrutura.

Tabela 1 - Comparação da acurácia das diferentes técnicas em relação ao as built. Adaptado de Popescu et al (2019)

\begin{tabular}{|c|c|c|c|c|c|c|c|}
\hline \multirow[t]{2}{*}{ Região } & \multirow{2}{*}{$\begin{array}{c}\text { As built } \\
\text { (mm) }\end{array}$} & \multicolumn{2}{|c|}{ Escaneamento a laser } & \multicolumn{2}{|c|}{ Fotogrametria } & \multicolumn{2}{|c|}{$\begin{array}{l}\text { Escaneamento } \\
\text { infravermelho }\end{array}$} \\
\hline & & $(\mathrm{mm})$ & $\% \Delta \mathrm{L}$ & $(\mathrm{mm})$ & $\% \Delta \mathrm{L}$ & $(\mathrm{mm})$ & $\% \Delta \mathrm{L}$ \\
\hline Vão & 6900 & 6928 & 0,41 & 7735 & 12,10 & 6958 & 0,84 \\
\hline $\begin{array}{c}\text { Largura } \\
\text { (superestrutura) }\end{array}$ & 3900 & 3904 & 0,10 & 4376 & 12,21 & 3926 & 0,67 \\
\hline
\end{tabular}

\subsection{INSPEÇÕES ROBOTIZADAS}

Uma inspeção robotizada de estrutura pode ser entendida como aquela em que os equipamentos ou recursos de inspeção são aplicados de forma autômata por um equipamento dotado de sistema de locomoção.

Robôs vem sendo aplicados a vários anos na inspeção de tubulações para reduzir o tempo despendido na atividade, prevenir riscos associados à espaços confinados, ambientes com toxidade e à possibilidade de colapso parcial ou global da estrutura e até mesmo para desenvolver ações de manutenção, como limpeza e reparo de vazamentos (MOSHAYEDI et al, 2019).

Moshayedi et al (2019) expõe como fatores necessários para que o robô desenvolva uma inspeção eficiente a adequação de seu tipo de movimentação ao tipo de inspeção desejado - considerando-se, por exemplo, sua habilidade de escalar, lidar com interseçções tipo " $T$ " ou "+" ou fazer curvas acentuadas com pequeno raio-, a precisão de seu movimento rotacional, sua estabilidade, a adequabilidade de seu tamanho ao espaço disponível, seu peso, sua capacidade de transposição de obstáculos, seus recursos de iluminação, sua autonomia na iniciação de funções, seu material, sua segurança e gestão de energia e a precisão das ferramentas embarcadas. Evidentemente, esses fatores podem se aplicar também ao uso de robôs na inspeção de outras estruturas, em adição aos desafios impostos por suas particularidades.

Ridao et al (2010) relatam a aplicação de robô na inspeção subaquática de uma barragem de concreto, quadro em que o equipamento foi utilizado para o mapeamento da estrutura com sonar, orientando-se e registrando dimensões através de sensores a laser. Os autores destacam a importância do georreferenciamento do robô durante a inspeção, considerandose as grandes dimensões da estrutura e suas experiências anteriores, datadas de 2002 - esse recurso permite a montagem de fotomosaicos da estrutura durante o processamento dos dados.

A montagem de fotomosaicos georreferenciados como produto da inspeção subaquática robotizada é relatada também por Hirai e Ishii (2019), cujo equipamento registra a estrutura através de fotografias contínuas. Os autores realizaram testes para avaliar a acuidade do registro das coordenadas registradas, verificando diferenças inferiores a 0,50 m entre a posição real e a posição medida - valor que pode ser aceitável na inspeção de uma barragem, ainda que seja excessivo para estruturas menores.

O mapeamento de fissuras em inspeções automatizadas com robôs é relatado por autores como Lim et al (2014) e Prasanna et al (2014), que aplicaram a técnica em pavimentos - a superfície predominantemente plana facilita a movimentação do equipamento. O uso de robôs facilita a aplicação de técnicas combinadas, como GPR (Ground Penetrating Radar), eco-impacto e imagens de alta resolução, conforme ilustra a figura a seguir. 

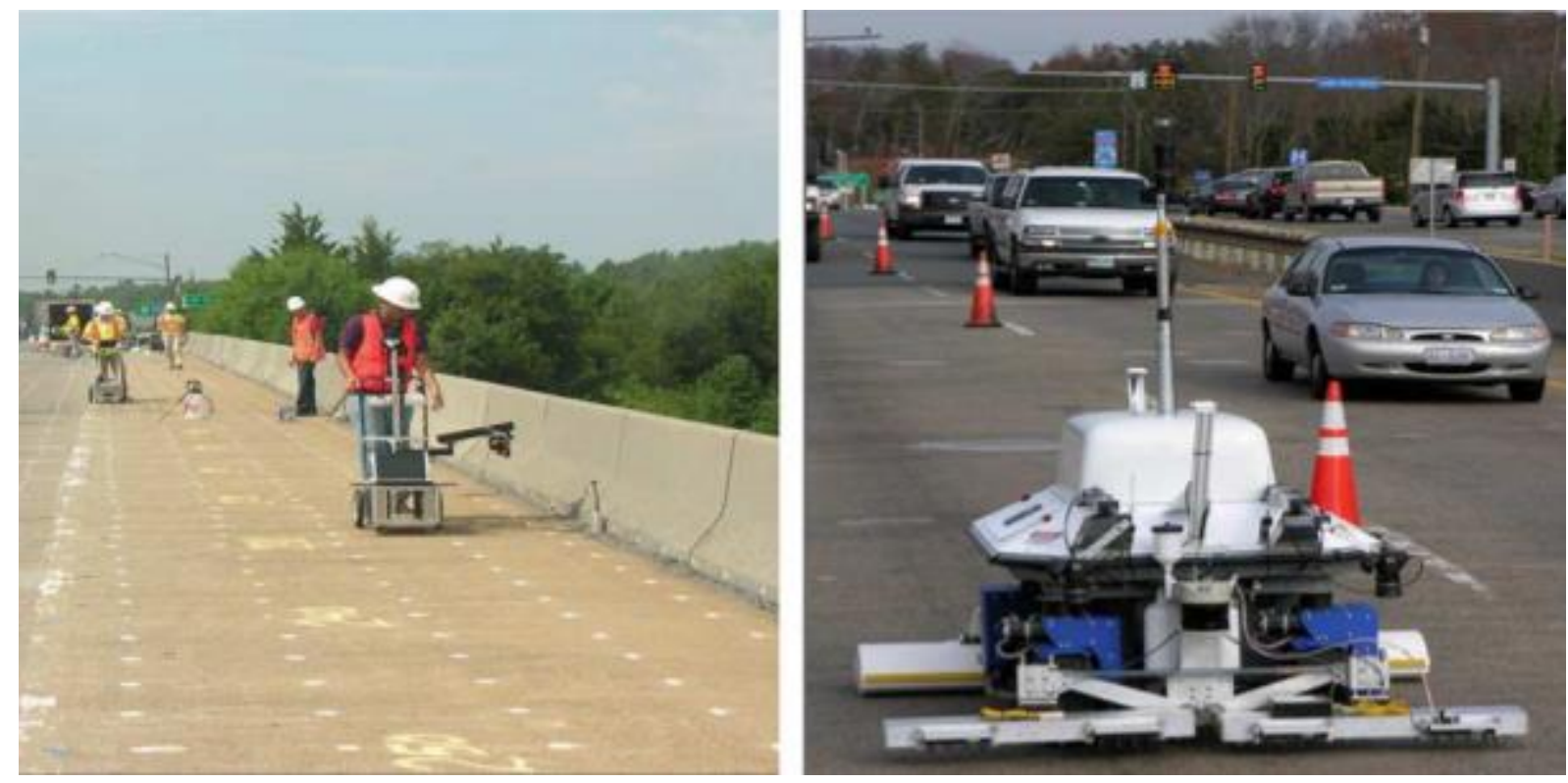

Figura 9: Inspeção robotizada do tabuleiro de uma ponte - coleta de dados por GPR (Ground Penetrating Radar), ecoimpacto e imagens de alta resolução (PRASANNA et al, 2014)

A menor distância entre a câmera e o alvo possibilitada pelo uso de robôs facilita a deteç̧ão de anomalias de pequenas dimensões, como fissuras de abertura inferior a $1 \mathrm{~mm}$, mas também pode gerar dificuldades a serem solucionadas através da aplicação de inteligência artificial e posterior análise do inspetor. Prasanna et al (2014) expõe que a presença de pequenas irregularidades na superfície, condição característica de compósitos como o concreto, representa desafios aos algoritmos, uma vez que é detectado um volume significativo de informações desnecessárias, como ilustra a figura a seguir.
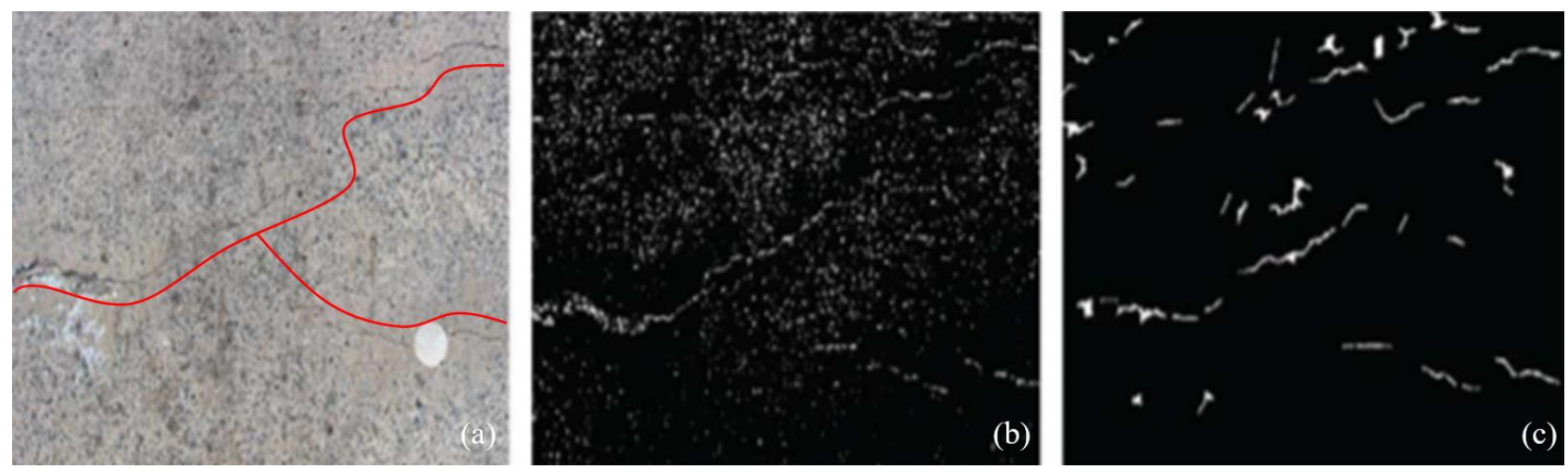

Figura 10: Deteç̧ão de fissuras em inspeção robotizada. Fissuras destacadas em vermelho - (a) - e imagem tratada em duas etapas - (b) e (c). Adaptado de Prasanna et al (2014)

Expõe-se, no entanto, que apesar do excesso de dados potencialmente prejudicar a identificação das anomalias relevantes - aqui consideradas como aquelas em evolução ou que representem risco à durabilidade, funcionalidade ou estabilidade do elemento estudado - em uma primeira campanha de inspeção, a reinspeção com a mesma técnica, utilziando equipamentos de alta acuidade e precisão, pode permitir a deteç̧ão automatizada de anomalias novas ou que sofreram aumento de sua criticidade. 


\section{CONSIDERAÇÕES FINAIS}

O presente artigo expôs o crescimento do interesse na aplicação de VANTs em inspeções, assim como o uso de técnicas como escaneamento a laser, escaneamento infravermelho e fotogrametria, abordando o produto decorrente e os desafios encontrados.

O uso de novas tecnologias na inspeção de estruturas apresenta diversas vantagens potenciais, como a possibilidade da coleta de dados com mais segurança e velocidade, assim como a implementação de recursos de deep learning para detecção de parte das anomalias. Trata-se de um ramo em crescimento, fruto da integração multidisciplinar entre a Engenharia Civil, a Tecnologia da Informação e a Cartografia. Verificou-se que as tecnologias abordadas no artigo apresentam alta aplicabilidade prática; contudo, elas devem ser entendidas como complementares à inspeção tradicional, não substituindo a necessidade de inspetores em campo, aptos a avaliar as estruturas com senso crítico, vistoriar áreas às quais os equipamentos não possuem pleno acesso, realizar análises tátil-visuais, limpezas, prospecções e ensaios.

No que se refere à análise dos dados, os recursos informatizados se mostram fundamentais para o adequado aproveitamento das nuvens de pontos, assim como das fotografias e filmagens em alta resolução. Os produtos desses trabalhos representam solicitações significativas para o hardware utilizado, podendo impor a necessidade de seccionamento de arquivos ou de balanceamento da densidade das nuvens de pontos e da resolução das imagens, adequando-os à real necessidade do inspetor.

Por fim, ressalta-se a necessidade da formação de extensos bancos de dados para o treinamento de algoritmos de inteligência artificial que possam prestar amplo apoio à detecção e classificação de anomalias em estruturas de concreto armado, mesmo na presença de manchas, sujidades e falhas no acabamento dos elementos - condição inerente às estruturas em uso a um longo período, especialmente em áreas urbanas e industriais -, dadas às limitações dos algoritmos aplicáveis atualmente, e a possibilidade de desenvolvimento de modelos preditivos da evolução do quadro patológico com base na repetida digitalização da estrutura ao longo do tempo.

\section{REFERÊNCIAS}

ASSOCIAÇÃO BRASILEIRA DE NORMAS TÉCNICAS. NBR 5674: Manutenção de edificações — Requisitos para o sistema de gestão de manutenção. Rio de Janeiro, 2012.

ASSOCIAÇÃO BRASILEIRA DE NORMAS TÉCNICAS. NBR 6118: Projeto de estruturas de concreto Procedimento. Rio de Janeiro, 2014.

ASSOCIAÇÃO BRASILEIRA DE NORMAS TÉCNICAS. NBR 9452: Inspeção de pontes, viadutos e passarelas de concreto - Procedimento. Rio de Janeiro, 2019.

DUQUE, Luis. UAV-based bridge inspection and computational simulations. Thesis (Master Degree). Electronic Theses and Dissertations. 2159. 168 p. 2017.

FEDERAL HIGHWAY ADMINISTRATION (FHWA). FHWA-HIF-19-056. Tech Brief - USE OF SMALL UNMANNED AERIAL SYSTEMS FOR BRIDGE INSPECTION. Outubro de 2019. Disponível em: https://www.fhwa.dot.gov/uas/resources/hif19056.pdf. Acessado em 20/12/2019.

HIRAI, Hiroyasu; ISHII, Kazuo. Development of Dam Inspection Underwater Robot. Journal Of Robotics, Networking And Artificial Life, [s.1.], v. 6, n. 1, p.18-22, 2019. Atlantis Press.

KIM, In-ho et al. Application of crack identification techniques for an aging concrete bridge inspection using an unmanned aerial vehicle. Sensors, [s.l.], v. 18, n. 6, p.1881-1895, 8 jun. 2018. MDPI AG. http://dx.doi.org/10.3390/s18061881.

LIM, Ronny Salim; LA, Hung Manh; SHENG, Weihua. A Robotic Crack Inspection and Mapping System for Bridge Deck Maintenance. Ieee Transactions On Automation Science And Engineering, [s.1.], v. 11, n. 2, p.367-378, abr. 2014. Institute of Electrical and Electronics Engineers (IEEE). 


\section{CBPAT 2020 \\ CONGRESSO BRASILEIRO DE PATOLOGIA DAS CONSTRUÇÕES \\ DE 15 A 17 DE ABRIL | FORTALEZA - CE \\ ISBN 978-65-86819-05-2}

LIU, W. et al. LiDAR-based bridge structure defect detection. Experimental Techniques, [s.1.], v. 35, n. 6, p.27-34, 1 jul. 2010. Springer Science and Business Media LLC.

MERAL, Cihan. Evaluation of laser scanning technology for bridge inspection. Thesis (Master Degree). Civil Engineering Department, Drexel University. 2011.

MOSHAYEDI, Ata Jahangir et al. Design and Development of Pipe Inspection Robot Meant for Resizable Pipe Lines. International Journal Of Robotics And Control, [s.1.], v. 2, n. 1, p.25-35, 10 jan. 2019. Sciedu Press.

POPESCU, Cosmin et al. 3D reconstruction of existing concrete bridges using optical methods. Structure And Infrastructure Engineering, [s.1.], v. 15, n. 7, p.912-924, 13 abr. 2019.

PRASANNA, Prateek et al. Automated Crack Detection on Concrete Bridges. Ieee Transactions On Automation Science And Engineering, [s.1.], v. 13, n. 2, p.591-599, abr. 2016. Institute of Electrical and Electronics Engineers (IEEE).

RIDAO, Pere et al. Visual inspection of hydroelectric dams using an autonomous underwater vehicle. Journal Of Field

Robotics, [s.1.], v. 27, n. 6, p.759-778, 13 jul. 2010. Wiley. 